Kap. 5 befasst sich mit einzelnen textuellen Phänomenen wie Sprechakten, Textkohärenz und Textsorten. Das Kapitel rechtfertigt sich aus dem zugrunde gelegten Syntaxbegriff, der sich nach Engel auf das Zusammenfügen sprachlicher Elemente zu immer größeren Einheiten bis hinauf zum Text bezieht (33). Kap. 6 gibt einen Überblick über Satzbauphänomene wie Kongruenz, Negation, Häufung und Apposition.

Zusammenfassend lässt sich formulieren, dass für den Leser, der die früheren Auflagen der Syntax der deutschen Gegenwartssprache nicht kennt, diese 4. Auflage eine sehr gut strukturierte, in einem leicht verständlichen Stil verfasste Arbeit darstellt. Der Band vermittelt wesentliches, aber auch hintergründiges Wissen über die Syntax des Deutschen. Ein weiterer positiver Aspekt des Buches äußert sich darin, dass Engel syntaktische Erscheinungen nicht nur in Satz-, sondern auch in Textumgebungen erklärt. Allerdings hätte man sich gewünscht, dass Übungen oder Arbeitsfragen eingebaut werden für diejenigen, die das Buch im Selbststudium benutzen wollten. Dies soll jedoch nicht den Wert dieser 4. Auflage der Syntax der deutschen Gegenwartssprache schmälern. Ob im Bereich der Wörter und ihrer Kategorisierung, des Satzes und seiner Definition, der Ergänzungen, ihrer Kategorisierung und der Benennung der Kategorien - der Band wirft grundsätzliche Fragen auf, die Anregungen für zukünftige Forschungen darstellen.

Studierenden, aber auch Lehrenden der Germanistik und des Deutschen als Fremdsprache, die sich vor allem mit valenztheoretischen Fragestellungen beschäftigen, ist das Buch nachdrücklich zu empfehlen.

\title{
Eppert, Franz:
}

Grammatik-ABC für Deutsch als Fremdsprache auf Zertifikatsniveau und Niveaustufen A1, A2, B1, B2. Ein kleines Handbuch für Lernende und Lehrende. Einfach - klar - praktisch. Frankfurt a. M.: R. G. Fischer, 2008. ISBN 978-3-8301-1194-8. 295 Seiten, $€ 22,80$

\section{(Claudia Bolsinger, Hamburg)}

Schon der Titel ist befremdlich: Welches Niveau ist denn nun angesprochen? Ist Zertifikatsniveau nicht das Gleiche wie B1? Wieso »klein«, wenn das Buch fast 300 Seiten umfasst und Din-A4-Format hat? Warum ist die Europakarte auf dem Einband schwarz auf dunkelblauem Grund?

Öffnet man den Band, setzt sich das Erstaunen fort: Was für ein gelbliches, dickes Papier wurde hier benutzt (das »kleine « Handbuch wiegt fast ein Kilo!)? Auch das Layout schreckt zunächst ab: Bleiwüste, kleine Schrift, keine Farben, keine Illustrationen. Einzige Gestaltungsmerkmale sind Fett- und Kursivdruck. Hat da jemand seinen Computerausdruck veröffentlicht? Oder ist man schon so abhängig 
von einer eingängigen, farblich differenzierten, es dem Auge leicht machenden Gestaltung, dass man dem Inhalt keine Chance mehr gibt? Sehen wir uns also den Inhalt an:

Im Vorwort referiert der Autor auf Lernzielbeschreibungen in Lehrwerken der Jahre 1972 bis 2001. Er gliedert in drei Abschnitte: Der erste Teil umfasst ein systematisches Verzeichnis von Wort-, Satz-, Text- und Sprachhandlungsgrammatik. Dieser Teil ist vor allem für Lehrende gedacht. Im zweiten Teil folgt das eigentliche Grammatik- $A B C$, das in alphabetischer Anordnung mit 141 Stichwörtern ein Grammatikverzeichnis für Lernende zur Verfügung stellen will. Der letzte Teil ist ein 350 Fachtermini umfassendes Suchregister. Etwas vollmundig klingt die Ankündigung im Vorwort »nicht versprochen, sondern verwirklicht«. Dahinter steht der Anspruch, ein sich selbst erklärendes, keine Fragen offen lassendes Grammatikkompendium der deutschen Sprache vorgelegt zu haben. »Kein einziges Fachwort bleibt unerklärt « dank eines redundanten Verweissystems, das umfangreiches Blättern vermeiden soll. Sehen wir hierzu ein Beispiel: Was sind Sätze? Dazu die Erklärung: »Sätze sind Kombinationen von $\rightarrow$ Satzgliedern $(\rightarrow$ Ergänzungen und $\rightarrow$ Angaben) und Wortgruppen ( $\rightarrow$ Attributen) auf der Basis von einfachen und komplizierten $\rightarrow$ Valenzen.«Einfach? Kein Weiterblättern? Für einen Lerner zwischen A1 und B2 zu verstehen?

Großen Wert legt der Autor auf die Darstellung von Sprachphänomenen und Regeln in Form von Tabellen. Sie - und sie allein - sollen die Übersichtlichkeit, Nachvollziehbarkeit und Verständlichkeit garantieren. Es gibt kaum eine Seite in diesem Werk, die keine Tabelle enthält, was vor allem Probleme mit der Zeilentrennung beim Seitenwechsel macht. Überhaupt stellt sich auch hier ein Selfmade-Eindruck ein: Die Formatierung ist durch falsches Einrücken und Trennen, doppelte Leerzeichen etc. geprägt.

Fazit: Wer - als Lehrender! - eine Regel, ein grammatisches Phänomen, ein Beispiel braucht, der findet hier, was er sucht. Aber er findet es auch an vielen anderen Orten, die einfacher $\mathrm{zu}$ handhaben und ansprechender gestaltet sind.

\section{Falk, Simone:}

Musik und Sprachprosodie. Kindgerichtetes Singen im frühen Spracherwerb. Berlin: de Gruyter, 2009 (Language, Context and Cognition). - ISBN 9783-11-021989-0. 340 Seiten, € 99,95

\section{(Ewa Wieszczeczyñska, Wrocław / Polen)}

Bei dem vorliegenden Buch handelt es sich um die Veröffentlichung der Dissertation der Autorin. Sie weist darauf hin, dass die prosodische Struktur der Sprache Kleinkindern in den ersten Stadien des Spracherwerbs behilflich sein könne, wichtige Aspekte ihrer Muttersprache zu beherrschen, und sie versucht in ihrer 